\title{
Enhancing Quality of Images using Fuzzy Logic and Singleton Parameters
}

\author{
J. B. Hayfron- Acquah, PhD \\ Senior Lecturer, Department of \\ Computer Science \\ Kwame Nkrumah University of \\ Science and Technology \\ Kumasi, Ghana
}

\author{
J. K. Panford \\ Senior Lecturer, Department of \\ Computer Science \\ Kwame Nkrumah University of \\ Science and Technology \\ Kumasi, Ghana
}

\author{
Y. Poakwah Gyimah \\ Department of Computer Science \\ Kwame Nkrumah University of \\ Science and Technology \\ Kumasi, Ghana
}

\begin{abstract}
There are a lot of images around carrying valuable information yet because of its poor quality, necessary information cannot be extracted. A low quality image is perceived to have low contrast and poorly defined boundaries between the edges. Most watched images speak to just a corrupted form of the first scene since genuine imaging systems and additionally imaging conditions are normally poor. The principal objective of this study is to manipulate and process an image so that it has a better presentation than the original image. When an image is processed for visual interpretation, the viewer, and the user is the ultimate judge of how well a method works. The visual evaluation of image quality is highly personal to the viewer and the user. The work concentrates on essentially the analysis of the visually enhanced images and a fuzzy approach for further enhancing these images, to make it more readable. The performance of the proposed technique was evaluated in terms of the visual quality, and the stability of the performance of the image enhancement techniques using eight image analysis parameters, to quantify the differences between the original image and the enhanced image. The experiment was carried out to study the performance of the image enhancement schemes and fuzzy logic image at different levels of image defections. The research analyzed this method with PSNR, MSE IQI, SI, and other important metrics in addition to visual comparison. The results then show this method as very good for reducing image defects.
\end{abstract}

\section{General Terms}

Image Preprocessing, Edge Detection, Median Filtering, Fuzzification

\section{Keywords}

Fuzzy logic, Gray Scale Image, Image Enhancement, Similarity Index, Standard Algorithm, Parameters

\section{INTRODUCTION}

Image enhancement fundamentally manages improving the impression of data in images for human viewers and giving 'better' contribution for other robotized image preparing methods. In this manner the chief goal of enhancing image is to change attributes of an image to make it more reasonable for a given errand and a particular spectator [1]. Images might be ruined by debasement, for example, linear frequency distortion, noise, and blocking relics. These wellsprings of corruption may emerge amid image catch or preparing, and have an immediate bearing on visual quality [2].

Images are required for some indispensable regions particularly biomedical image investigation, dynamic and activity scene examination, machine vision, remote sensing, and self-governing route. There are a lot of images around carrying valuable information but cannot be extracted because of their poor quality of which maybe vital for these regions. Again a gigantic piece of recorded pictures meets underhandedness effect of poor refinement because of absence of light amidst image obtaining, mistakenly setting of opening size and screen speed likewise nonlinear image intensities mapping. In this manner it is imperative to improve the image quality using certain kind of image enhancement procedures which are the principal task associated in image preparing to upgrade interpretability and appearance of the image [3].

Fuzzy logic has been stretched out to handle the idea of incomplete truth, where reality quality may run between totally genuine and totally false. It has ended up being a superb -decision for some control framework applications since it emulates human control logic. It doesn't require exact inputs; it's innately powerful, and can prepare any sensible number of inputs [4]. Hybrid systems joining fuzzy logic and standard algorithms are demonstrating their adequacy in a wide assortment of true issues [5]. Most keen procedures have specific computational properties (e.g. capacity to learn, clarification of choices) that make them suited for specific issues and not for others. For instance, while neural networks are great at recognizing patterns, they are bad at clarifying how they achieve their choices. Fuzzy logic systems, which can prevail upon loose data, are great at clarifying its choices yet can't consequently get the standards they use to settle on those choices. These restrictions have been a focal main impetus behind the formation of clever hybrid systems where two or more procedures are joined in a way that conquers the impediments of individual methods. To upgrade images that are degraded, artificial intelligence methods situated in fuzzy logic and standard image enhancement will be connected together. The motivations to consolidate these two standards leave the challenges and intrinsic impediments of each disengaged model [6].

The central aim of this research is to enhance degraded images using fuzzy approach. The study therefore aims image preprocessing techniques. Specifically the study aims to:

- propose an algorithm and design a software application to enhance images using the standard image enhancement techniques and the fuzzy logic techniques

- test the designed technique to enhance some degraded, low contrast images into a more readable form.

- apply image analyses and measurement parameters to compare the level of improvement in the images after and 
before fuzzy logic techniques.

\section{JUSTIFICATION OF STUDY}

A low quality image is perceived by low contrast and poorly defined boundaries between the edges. Most watched images speak to just a corrupted form of the first scene since genuine imaging systems and additionally imaging conditions are normally poor. From [7], different sorts of degradations (geometric and also radiometric) are brought into the image during the acquisition by such elements as imaging geometry, lens deviation, wrong focus, movement of the scene, systematic and random sensor errors, and so forth. Image enhancement can be considered as change of low quality image into great quality image to make its significance clearer for human discernment or machine examination [8]. The use of fuzzy methodology in upgrading quality images will help with the end goal of making the subject of the image look superior to the camera and lighting without anyone else's input could catch in the wake of experiencing change. Also, this exploration will contribute information to the current learning in the field of images all the more particularly image enhancement and neuro-fuzzy methodology.

\section{SOME FIELDS IMAGE}

\section{ENHANCEMENT HAS CONTRIBUTED}

Image Enhancement has contributed to research advancement in a variety of fields of which some include:

\subsection{Astrophotography}

This field confronts challenges because of light and noise contamination that can be minimized by image improvement [9]. For continuous sharpening and contrast enhancement a few cameras have in-fabricated image enhancement capacities. Additionally, various software [10] [11], permit altering such images to give better and striking results.

\subsection{Air Sciences}

In air sciences [12] [13] [14] image enhancement is utilized to decrease the impacts of cloudiness, haze, fog and turbulent climate for meteorological perceptions. It helps in identifying shape and structure of remote items in environment detecting [15]. Satellite images experience image restoration and improvement to eliminate noise.

\subsection{Oceanography}

In this field the investigation of images uncovers intriguing features of water flow, sediment concentration, geomorphology, and bathymetric models to give some examples. These elements are all the more unmistakably discernible in images that are digitally improved to beat the issue of moving targets, lack of light and blur environment.

\subsection{Legal Sciences}

In legal sciences [16] [17] [18] picture improvement is utilized for ID, proof social occasion and observation. Pictures acquired from unique finger impression discovery, security recordings examination and wrongdoing scene examinations are upgraded to help in recognizable proof of offenders and assurance of casualties.

\subsection{Medical Imaging}

Medical imaging [19] [20] [21] utilizes picture upgrade strategies for lessening commotion and sharpening subtle elements to enhance the visual representation of the image. Since moment subtle elements assume a basic part in conclusion and treatment of sickness, it is vital to highlight imperative components while showing medicinal images. This makes image enhancement a fundamental supporting instrument for survey anatomic ranges in MRI, ultrasound and $\mathrm{x}$-rays and so on.

\section{METHODOLOGY}

This study aims to develop a scheme to manipulate and process an image so that it has a better presentation than the initial image. This section entails the system design procedures of the proposed algorithm. The algorithm is proposed and implemented to enhance images using fuzzy technique. The proposed algorithm is to avoid the problem that existing techniques of image enhancement are not effective in dealing with imprecise data, vague and uncertain information. It provides satisfactory results in low contrast and light variations areas. The proposed methodology has following general steps:

Step 1: Input to the system, an original image. No calculation is done in this step. The image is shown in pre-defined axes.

PHASE ONE: Image Preprocessing.

This phase extracts informational features about the image before processing. These extracted features are used to determine if the image needs further processing or not.

Step 2: The colour of image is identified. If it is gray scale image, then image gradient is applied on the image and saved or written to the computer storage. An image gradient is a directional change in the intensity or color in an image. Image gradients are used to extract information such as the features of the images which will be used for the step 4 .

Step 3: If the image is coloured then it is divided into three channels, that is, its Red, Green and Blue Channels and saved or written individually to the computer storage. Again, then, the image gradient is applied on each channel to extract information such as the features of the images which will be used for the step 4 .

Step 4: The features of the image are extracted from it to check for the level of distortion that is rendering the image not readable or useful for further processing. This, which is done by the system, using conversion to image gradient, can also be done by the human user by simply describing the image visually, as not clear, not bright, dark or having a uniform type of noise.

\section{PHASE TWO: Standard Image Enhancement.}

This face is optional and could be omitted by the human user if the user does not want to enhance the edges or noise filtered images, but wants to go straight to the fuzzy enhancement processing.

Step 5: This step is user and result dependent. It is optional and can be omitted if the user sees no reason to enhance the image using any standard image enhancement techniques. After applying image gradients on the image, the human user can apply different image enhancement models made available by the system and which is under study. The human user can enhance the image using the edge detection algorithm or filter some noise spectrums within the image using the center-weighted median filtering algorithm. The user can also add some random Gaussian or salt-and-pepper noise if the image inputted carries no uniform type of noise generated by a third-party image editing software such as Adobe ${ }^{\circledR}$ Photoshop or dotPDN® Paint.Net.

PHASE THREE: Fuzzy Image Processing.

Step 6: Fuzzy sets are applied on pixels to normalize the 
appearance of these pixels and prepare the image for fuzzy image enhancement. Fuzzy-based pixel normalization provide promising result in image processing by calculating the average brightness and darkness values in some neighborhood $m x n$ of the original image and replaces the gray level with an average value. The brightness or darkness averaging operation adjusts and stretches the image contrast, as well as sharpens and preserve the edges of the image.

\section{PHASE FOUR: Image Enhancement Measurement}

Step 7: Quantify the improvement in the fuzzy-based enhanced image by comparing this enhanced images of fuzzy inference mechanism with the other standard enhanced images or the original image.

This is illustrated by Figure 1 which depicts the overall basic design procedure of the Image Enhancement Technique using Fuzzy-Logic.

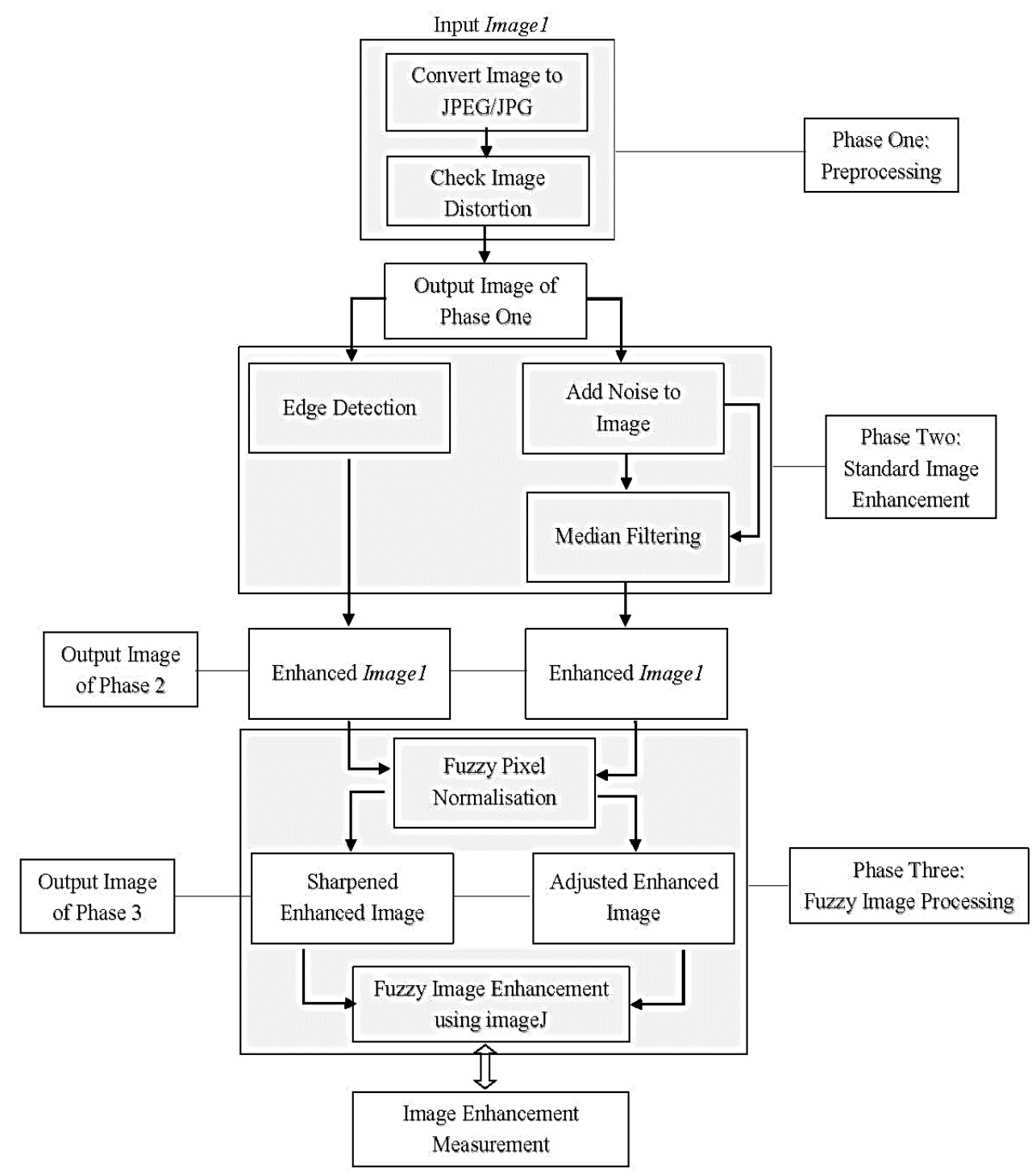

Figure 1: Design procedure of the Fuzzy-Based Image Enhancement Technique

\section{RESULTS}

As insinuated earlier, image enhancement plays an important role in digital image processing. Image enhancement transforms digital images to enhance the visual information which is a primary operation for almost all vision and image processing tasks in several areas such as Computer vision, biomedical image analysis and other fields.

\subsection{RO1: Proposed Mamdani Fuzzy Logic Technique}

The mapped values are classified into three fuzzy partitions (regions) DARK, GRAY, and BRIGHT as shown in Figure 2. Out of the range [0 255], the DARK region covers the range [0 127] where the maximum range value at which DARK attains a true characteristic $(M F=1)$ is 70 . This sets the DARK Params [0 70 127]. Again, to continue, the GRAY region covers a range [57 184] where the maximum range value at which GRAY attains a true characteristic $(\mathrm{MF}=1)$ is 127 . This divides the range of the GRAY region to two which are [57

127] and [127 184]. This also sets the GRAY Params [57 127 184]. Further, the last region which covers the rest of the pixel values, BRIGHT region covers a range [123 255] where the maximum range value at which BRIGHT attains a true characteristic $(\mathrm{MF}=1)$ is 180 . This divides the range of the BRIGHT region to two which are [123 180] and [180 255]. Combining these two given ranges, set the overall range parameters of BRIGHT to Params [123 180 255] as illustrated in figure 2. 


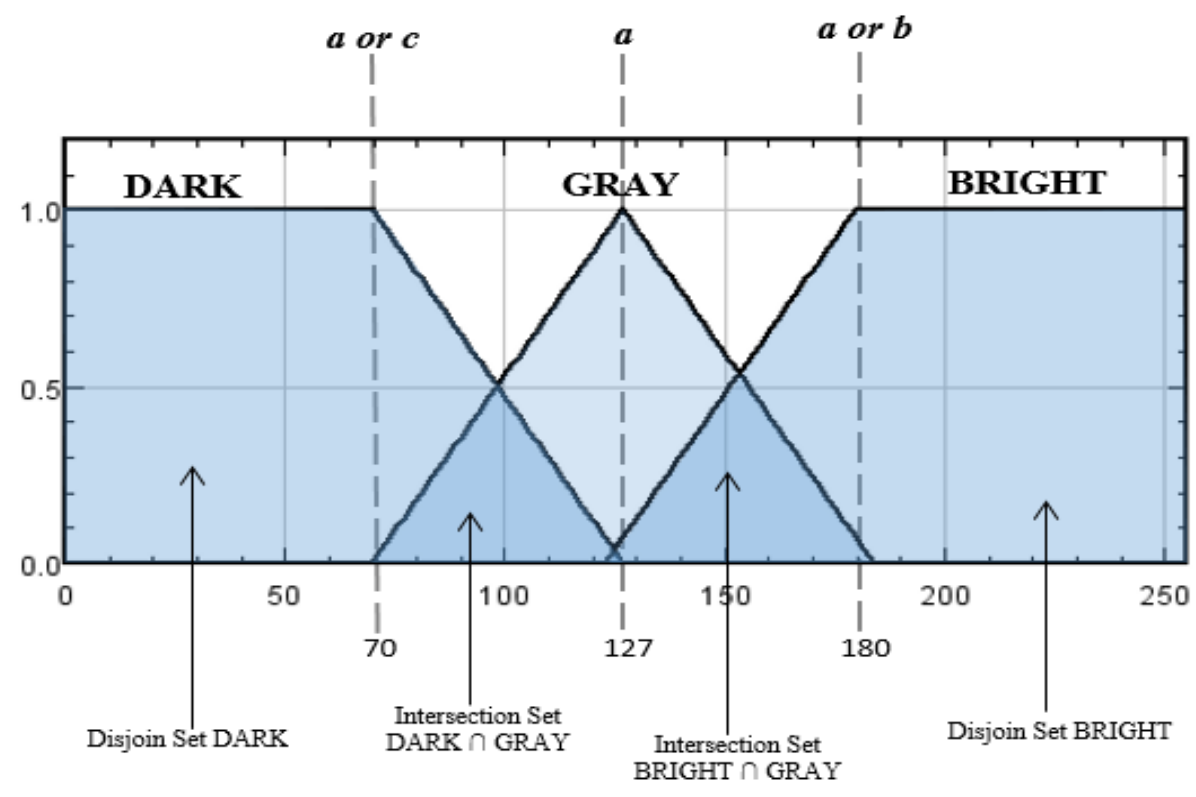

Figure 2: Fuzzy partitions (regions) DARK, GRAY, and BRIGHT

\subsection{RO2: Test Proposed Technique on Degraded Image}

\subsubsection{Option 1: Edge Detection}

The detection of object boundaries is one of the most fundamental problems in image understanding and computer vision. The importance of high-contrast lines that outline objects was clearly concentrated in this algorithm. In fact, the algorithm used an image filter function in

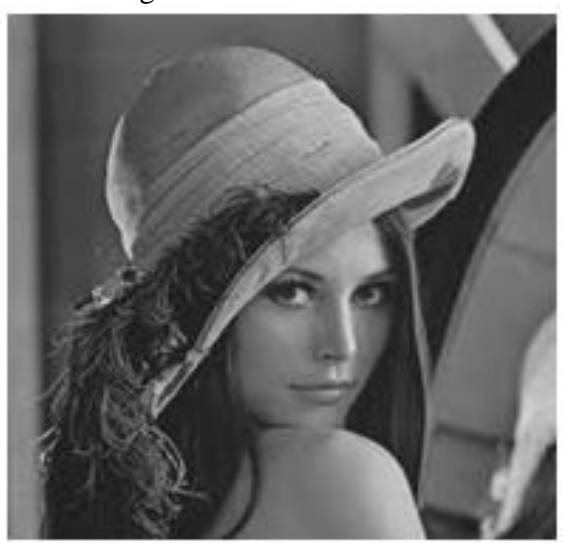

(a)
MATLAB®R2016a, medfilt2, which performs median filtering of an image matrix in two dimensions. medfilt 2 pads the image with 0's on the edges, so the median values for points within the contour edges of a given image might appear appealing. This idea, specifically detect black lines against a white background or white lines against a dark background, or simply light-dark discontinuities in the intensity as shown in figure 3 .

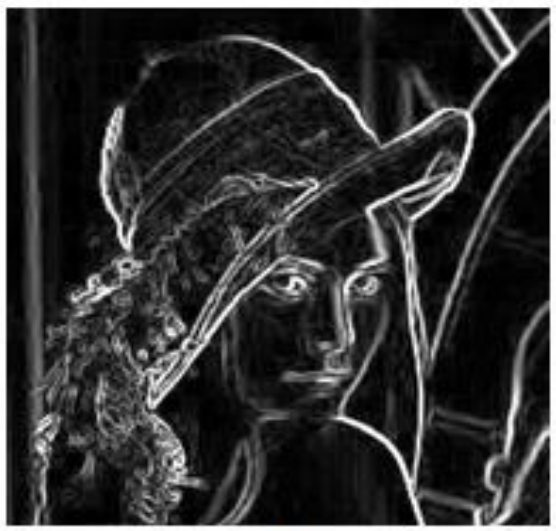

(b)

Figure 3: (a) Original Image, (b) Edge Detected image Source: Author's Proposed Algorithm

[22] proposed the method in which inversion of image is used and black pixels are converted into white pixel and white into black. They further selected threshold in an image using fuzzy set theory. They added this technique to edge detection. Figure $4 \mathrm{~b}$ for instance, it was realized that denser areas were at the left of the image where the child's hair slightly covers her eyes or better still, her shoulders, where there are more lines in her dress. Left pixels are therefore not visually friendly and readable but figure $4 \mathrm{c}$ presents visually and readable denser areas. 


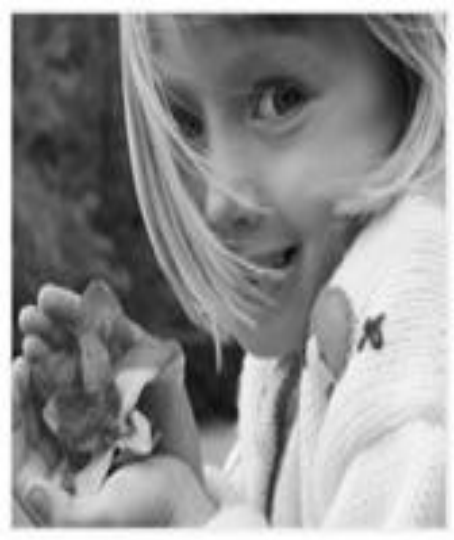

(a)

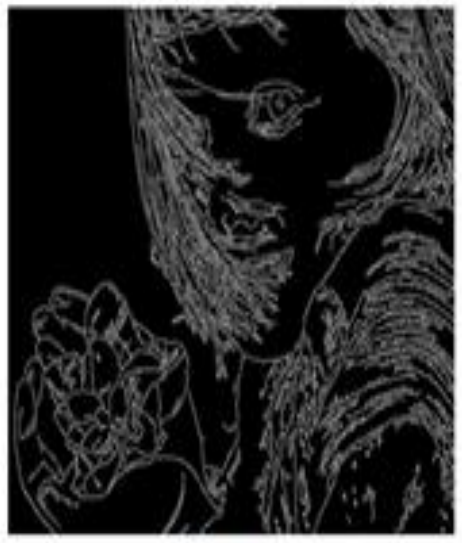

(b)

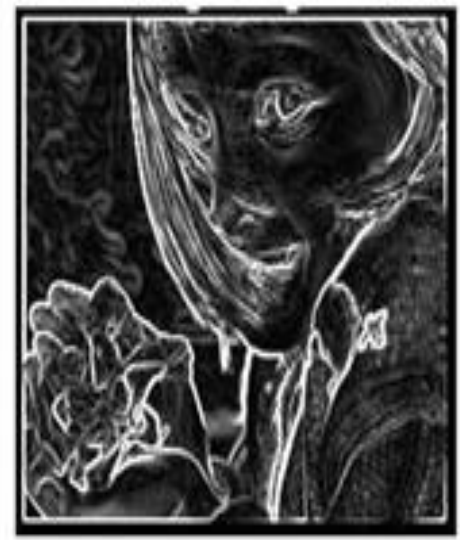

(c)

Figure 4: (a) Original image, (b) [22], (c) Proposed Technique

\subsubsection{Option 2: Filtering}

The proposed technique used Centre Weighted Median Detection which computes the median over the surrounding neighborhood; and replaces the pixel value with the weighted median). [23] proposed another technique for enhancement of

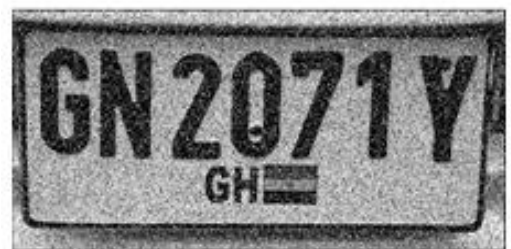

(a) image from noise using $\gamma$ (Gamma) correction median filtering which is based on finding the statistical operation of finding the median of an image with noise. Figures 5 and 6 show the results of these techniques.

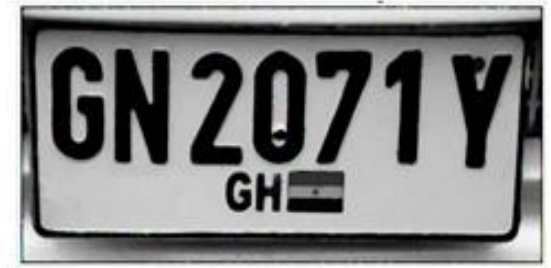

(c)

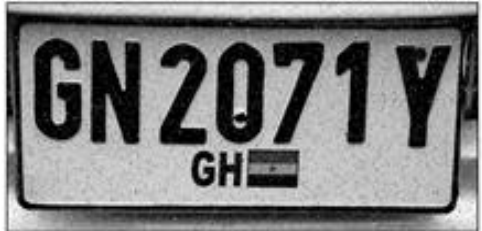

(b)

Figure 5: (a) Original image, (b) [23], (c) Proposed Technique

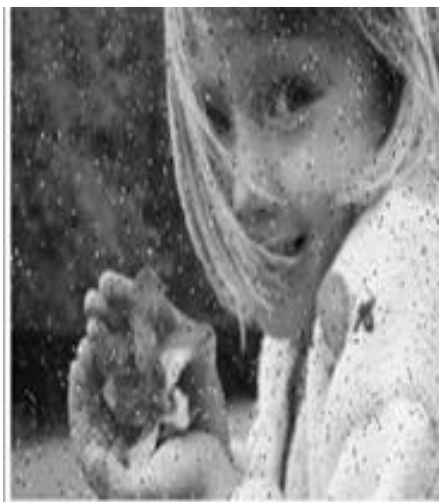

(a)

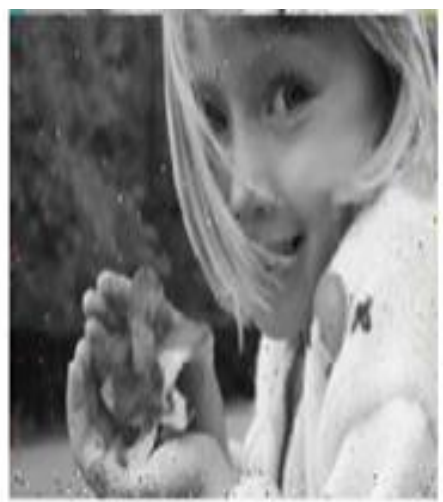

(b)

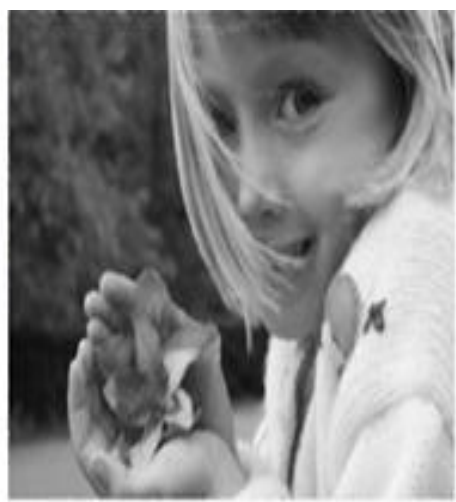

(c)

Figure 6: (a) Original image, (b) [23], (c) Proposed Technique 


\subsubsection{Fuzzy Image Processing}

To achieve a reliable and perfect software system for fuzzybased image enhancement, in this section, the implementation of the improved image enhancement algorithm is used to filter out noise, heighten image visibility, and enhance edges of the contents within an image. Additionally, the enhanced image is passed through a fuzzification engine to further bring out the desired contents within the image. The first idea was to maximize the appearance of a scanned document page and also, increase the quality of this scanned image for further processing such as in an Object Character Recognition (OCR). The scanned image is an 8-bit gray scale image. Dark patterned fragments of $75 \%$ defects are added to the scanned image. Figure 7 then depicts the results after the poor scanned book went through the process of the proposed technique.

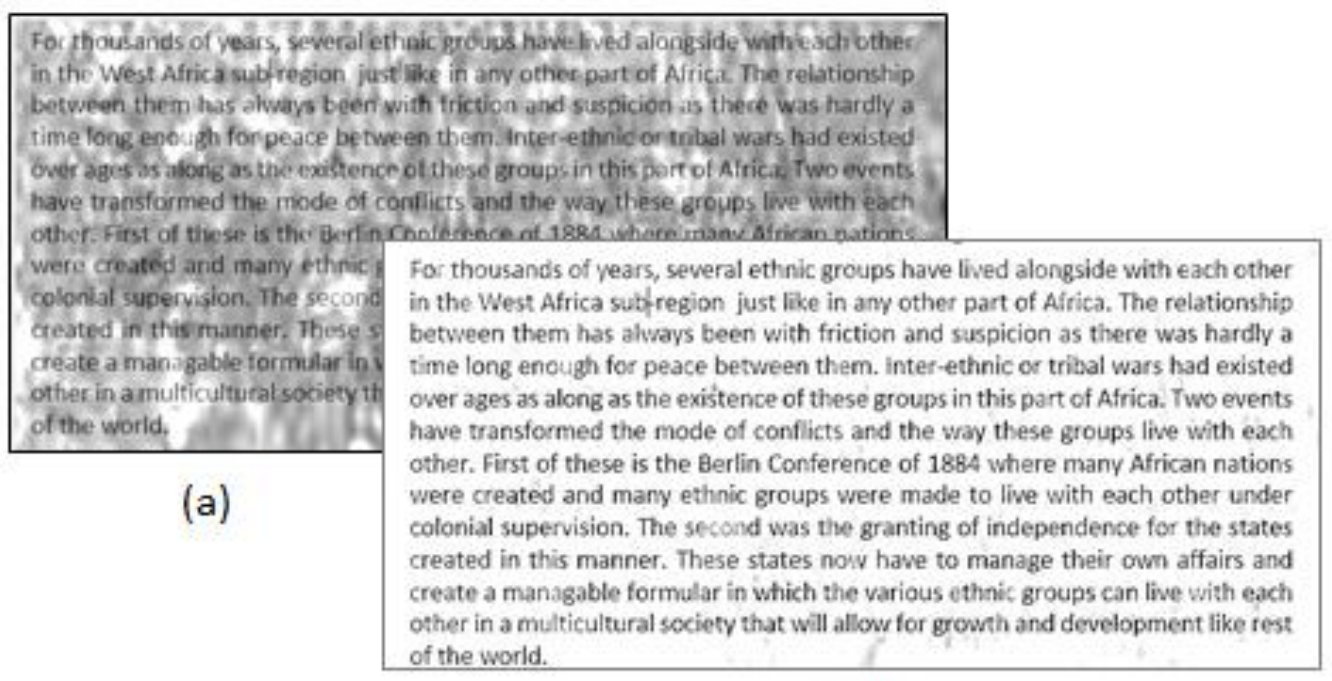

(b)

Figure 7: (a) Poor scanned book, (b) Enhanced scanned book (Using the Proposed Technique)

\subsection{RO3: Compare Image Enhancement Levels}

In order to evaluate the image enhancement results obtained from different methods and compare the methods with the final fuzzy based image enhancement, the image assessment measures and parameters are employed. The value of each quality assessment parameters of all mentioned image enhancement approaches are depicted in Table 1.

Table 1. Comparative results in image analysis parameters of different images before and after fuzzy enhancement

\begin{tabular}{lllllllll}
\hline \multirow{2}{*}{ Parameters } & test_image_1.jpg & test_image_2.jpg & \multicolumn{2}{c}{ test_image_3.jpg } & \multicolumn{2}{c}{ test_image_4.jpg } \\
\cline { 2 - 9 } & Before & After & Before & After & Before & After & Before & After \\
\hline MSE & 3394.18 & 9417.18 & 3135.10 & 9874.10 & 3698.2 & 9768.2 & 3107.7 & 9881.7 \\
RMSE & 58.2596 & 97.0422 & 55.9920 & 99.3685 & 60.8128 & 98.8342 & 55.7467 & 99.4067 \\
PSNR & 85.18 & 96.18 & 78.10 & 95.10 & 78.2 & 94.2 & 82.7 & 97.7 \\
MAE & 49.18 & 96.18 & 55.10 & 96.10 & 54.2 & 87.2 & 54.7 & 90.7 \\
SNR & 14.18 & 30.18 & 15.10 & 25.10 & 8.2 & 26.2 & 10.7 & 23.7 \\
IQI & 57.18 & 81.18 & 59.10 & 84.10 & 56.2 & 82.2 & 54.7 & 89.7 \\
SI & 42.82 & 18.82 & 40.90 & 15.90 & 43.80 & 17.80 & 45.30 & 10.30 \\
r & 0.5338 & 0.7808 & 0.5773 & 0.7859 & 0.6181 & 0.7479 & 0.6246 & 0.7362 \\
\hline
\end{tabular}

The experimental results show that fuzzy logic based image enhancement approach provides better performance when compared to the standard image enhancement for all the test images (test_image1 to test_image4). Image Quality Index (IQI) which is the differences and between reference and enhanced images are high for test_image4 (IQI=89.7\%), followed by test_image2 (IQI=84.10\%), then by test_image3 $(\mathrm{IQI}=84.10 \%)$, and lastly by test_imag14 (IQI=81.18\%). The Similarity Index (SI), being the differences between reference and enhanced images are lower for test_image4 (SI=10.30\%), followed by test_image2 (SI=15.90\%), then by test_image3 (SI=17.80\%), and lastly by test_imag14 (SI=18.82\%). These values when compared to values obtained from standard image enhancement are higher. The other evaluation measures like root mean square error (RMSE) were with higher values and the peak signal to noise ratio (PSNR) as well as the Correlation Coefficient (r) were also with higher values obtained for fuzzy based image enhancement approach are, therefore, comparatively better than the standard image enhancement approach proposed. So it is concluded that results obtained from the implementation of fuzzy logic based image enhancement approach performs better for all the test image cases and since fuzzy based image enhancement shows better performance further investigation is needed to improve this method.

\section{CONCLUSION}

The performance of the proposed algorithms was evaluated in terms of the visual quality, and the stability of the performance of the image enhancement techniques using eight image analysis parameters, to quantify the differences between the original image and the enhanced image. The experiment was carried out to study the performance of the image enhancement schemes and fuzzy logic image at different levels of image defections. The researcher analyzed this method with PSNR, MSE IQI, SI, and other important metrics in addition to visual comparison. The results then showed this method as very good for reducing image defects.

This work has not considered any image acquisition technique by the use of external image capturing device like the picture camera device. The work however concentrated only in the importation of images from a secondary storage device like a pendrive or an HDD or SDD. Therefore, image acquisition procedures can be inculcated into the image input steps so that the project could capture an image and instantly enhance the 
image using an optimistic enhancement technique.

\section{ACKNOWLEDGEMENTS}

Thanks go to the Omnipotent God for his wisdom, protection, guidance, and blessings towards us for the completion of this work.

\section{REFERENCES}

[1] Raman Maini \& Himanshu Aggarwal (2010). A Comprehensive Review of Image Enhancement Techniques. Journal of Computing, 2: 8

[2] Niranjan Damera -Venkata, Thomas D. Kite, Wilson S. Geisler, Brian L. Evans, \& Alan C. Bovik. (2000). Image Quality Assessment Based on a Degradation Model, IEEE Transactions on Image Processing, 9(4): 636

[3] Pandey A. K., Kavita A., \& Mohd. H. (2015). A Hybrid Approach for Enriching Image using Mamdani NeuroFuzzy Technique and its Comparative Analysis. International Journal of Computer Applications, Vol. 121

[4] Mahashwari $T$ \& Asthana A. (2013). Image Enhancement Using Fuzzy Technique International Journal of Research Review in Engineering Science \& Technology, vol. 2,ISSN 2278-6643.

[5] Fullér Robert. (1995). Neural Fuzzy Systems. ISBN 951650-624-0, ISSN 0358-5654, 208

[6] Vieira José, Fernando Morgado Dias, Alexandre Mota. (2004). Neuro-Fuzzy Systems: A Survey, 5th WSEASNNA International Conference on Neural Networks and Applications, Udine, Italia.

[7] Jan Flusser \& Tomáš Suk (1998). Degraded Image Analysis: An Invariant Approach. IEEE Transactions on Pattern Analysis and Machine Intelligence, Vol. 20, No. 6

[8] Mahashwari T \& Asthana A. (2013). Image Enhancement Using Fuzzy Technique International Journal of Research Review in Engineering Science \& Technology, vol. 2,ISSN 2278-6643.

[9] Fridman P. (2001). "Radio Astronomy Image Enhancement in the Presence of Phase Errors using Genetic Algorithms," in Int. Conf. on Image Process., Thessaloniki, Greece, pp. 612-615.

[10] Astroart, (2007). "Astroart 4.0," http://www.msbastroart.com/.

[11] Astrostack, 2007. "Astrostack 3.0," http://www.astrostack.com/.
[12] Armitage D. W. \& Oakley J. P. (2003). "Noise Levels in Colour Image Enhancement," in Visual Inform. Eng., London, UK, pp. 105-108.

[13] Buckingham J. M. \& Bailey J. (2003). "Imagery Enhancement to Meteorological Collection Platform," in Proc. Syst. And Inform. Eng. Design Symp., Charlottesville, VA, pp. 275- 281.

[14] Woodell G., Jobson D., Rahman Z. U. \& Hines G. (2006). "Advanced Image Processing of Aerial Imagery," in Proc. SPIE Visual Inform. Process. XIV, Kissimmee, FL, p. 62460E.

[15] Vorobel R. (1996). "Contrast Enhancement of RemotelySensed Images," in 6th Int. Conf. Math. Methods in Electromagnetic Theory, Lviv, Ukraine, pp. 472-475.

[16] Teddy K. (2002)."Fingerprint Enhancement by Spectral Analysis Techniques," in 31st Applied Imagery Pattern Recognition Workshop, Washington DC, WA, pp. 16-18.

[17] Greenberg S., Aladjem M., \& Kogan D. (2002). "Fingerprint Image Enhancement using Filtering Techniques," Real-Time Imaging, vol. 8, no. 3, pp. 227 236.

[18] Z. Abu-Faraj, A. Abdallah, K. Chebaklo, and E. Khoukaz. (2000). "Fingerprint Identification Software for Forensic Applications," in 7th IEEE Int. Conf. Electronics, Circuits and Systems, Jounieh, Lebanon, pp. 299- 302

[19] Tsai D. Y., Yongbum L., Sekiya M., Sakaguchi S. \& Yamada I. (2002). "A Method of Medical Image Enhancement using Wavelet Analysis," in 6th Int. Conf. Signal Process, Beijing, China, pp. 723-726.

[20] Haiguang C. A., Li Kaufman L. \& Hale J. (1994). "A Fast Filtering Algorithm for Image Enhancement," IEEE Trans. Med. Imag., vol. 13, no. 3, pp. 557-564.

[21] Ping W., Li J., Lu D., \& Ga C. (2006). "A Multi-scale Enhancement Method to Medical Images Based on Fuzzy Logic," in IEEE Region 10 Conference TENCON, Hong Kong, China, pp. 1-4.

[22] Knee B., W. (2011). Application of improved edge detection algorithm using canny overlapping method Journal of Applied Optics, 4(32), 678-682.

[23] Asadi, B. K., \& Amiri J. B. (2015). A review paper: noise models in digital image processing. arXiv preprint arXiv:1505.03489. 